\title{
Complex Situations Simulation When Testing Intelligence System Knowledge Base
}

\author{
Yu.Nechaev, A.Degtyarev, A.Boukhanovsky \\ Institute for High Performance Computing and Data Bases, Fontanka 118, 198005 \\ St.Petersburg,Russia \\ (int, deg, avb) @fn.csa.ru
}

\begin{abstract}
Construction of tool system for testing dynamic knowledge base of intelligence system (IS) of dynamic object (DO) behavior analysis and forecast is discussed. The system provides generation of environment and dynamics of floating DO - environment interaction in various operation conditions. Results of imitating experiment are given.
\end{abstract}

\section{Introduction}

Hardware and software design providing testing of knowledge base (KB) represents one of the important directions of the complex approach in development of intelligence technologies. At modern tool design the tendency of intellectualization in a direction of artificial intelligence methods application is precisely looked through. The analysis of existing tools show, that the main share of capacity and intellectuality of such toolkit is not associated with its architecture, but with functionalities of separate component of technological environment [1-4].

Packages APT KEE and G2 are among the most powerful and advanced tool systems. System G2 of firm Gensym is further development of system PICO and one of the most powerful environments for real-time systems. Due to openness of the interface and support of a wide spectrum of computing platforms system G2 allows to unite isolated automation means in a uniform complex control system. At the same time G2 is poorly adapted to perception of a complex information stream by development and testing of onboard integrated real-time systems. The tool system offered in the paper represents integrated component for the solution of a wide range of problems of ship's dynamics and offshore structures.

Novelty of the developed program technology consists in the following:

- the model of sea three-dimensional waves and wind in small-scale and synoptic ranges of variability is developed;

- the model of interaction of floating DO with an environment in various conditions of operation is developed;

- imitating experiment for DO dynamic characteristics assesment in extreme situations is carried out. This experiment is connected with loss of stability of oscillatory movement at a various level of external actions.

Principal difference of the tool system from other similar systems consists in simulation of a real picture of exerted actions. It provides reliability of the practical 
recommendations, which are given out by intelligence system in realization of inferential mechanism.

\section{Architecture and functionalities of system.}

The complex of the theoretical and practical questions connected with development of tools was considered in work [1]. Continuation of researches on perfection of the approach to system creation has allowed to formulate and realize technology of transforming the information when estimating DO behavior at any level of external actions.

The architecture and base components of the system are given in fig. 1 .

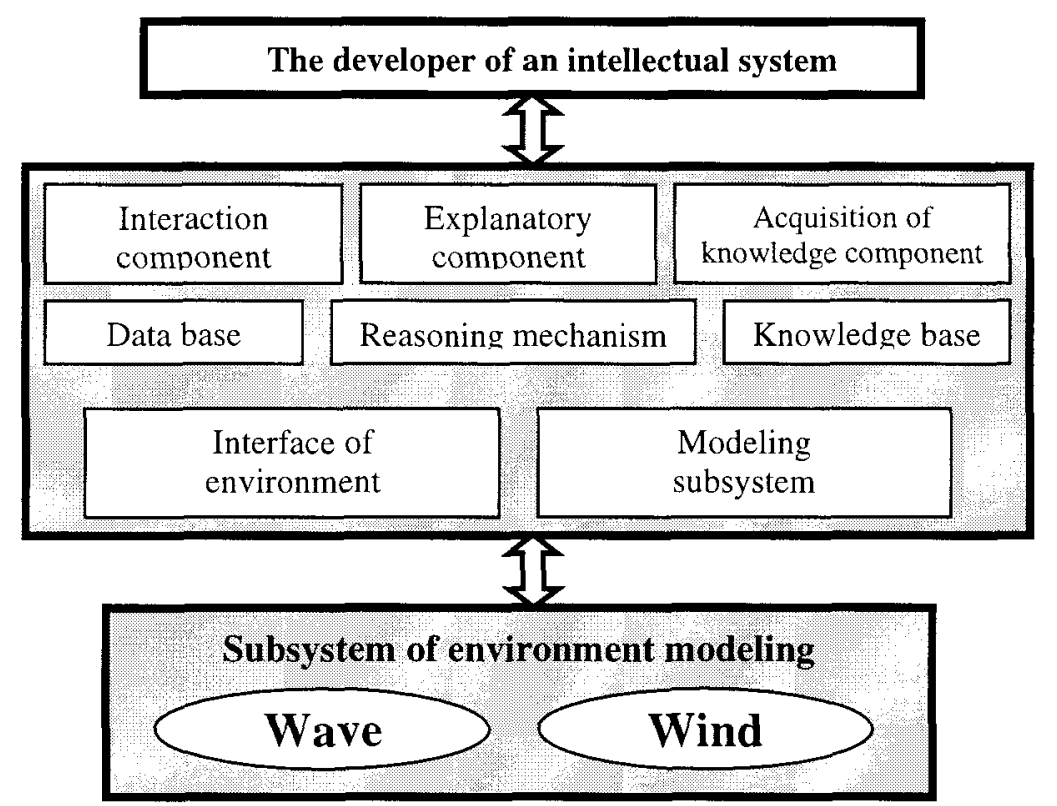

Fig. 1. Instrumental tool for knowledge base testing

The submitted structure includes traditional IS components (KB, database, solver, means of knowledge explanation and purchase) and the special complexes allowing the developer to interact with a subsystem of environment and object dynamics modeling. The basic principles fixed in a basis of system provide:

- technology of open systems, adaptability and dynamism;

- search of the decision based on fuzzy models of knowledge representation and imitating modeling;

- application of cognitive graphics and analysis of dynamic stages.

The testing problem is represented by the tuple:

$$
<H_{i}, S_{j}, T_{k}>
$$




$$
(i=1, \ldots, n ; j=1, \ldots, m ; k=1, \ldots, l)
$$

where $H, S, T$ are sets of hypotheses, symptoms and tasks.

Initial data (1) characterize matrixes:

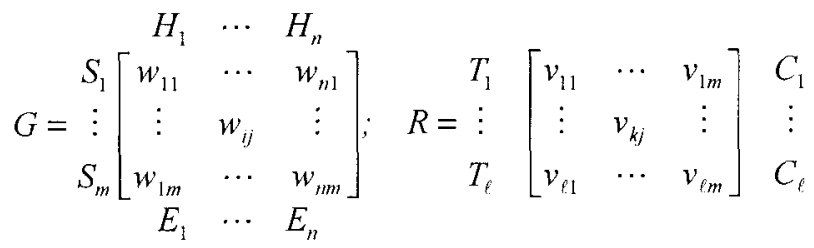

Here $E_{i}(i=1, \ldots n)$ are estimations; $C_{k}(k=1, \ldots l)$ are expenses; $w_{i j}(i=1, \ldots, n$; $j=1, \ldots, m)$ is weight of a symptom in the given hypothesis; $v_{k j}(k=1, \ldots, l ; j=1, \ldots, m)$ is characteristic vector specifying correspondence between a problem and symptoms.

In that specific case, at unequivocal correspondence between symptoms and problems matrix $R$ is identity. Then the initial data can be described by replacement of appropriate tasks in matrix $R$ and adding a vector-column of expenses on the right. Procedures of IS testing and diagnostics are based on application of acceptance decisions mechanisms with use of KB fuzzy models.
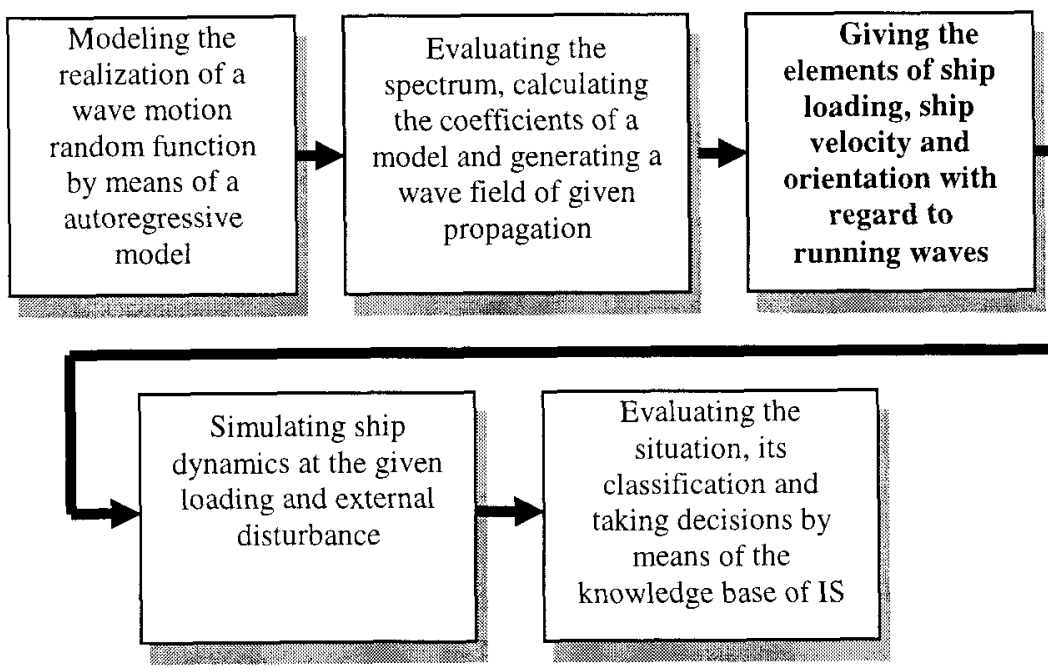

Fig. 2. Information flow in IS knowledge base using imitation modeling methods

\section{Specific features of external actions modeling}

The basic external actions influencing floating DO are determined by the irregular hydrodynamic forces of wind and wave character caused by surface wind, wind waves or swell, and also by fetch current. The integral property of these 
hydrometeorological processes is their space and time domain variability caused by superposition and interaction of a large number of factors. Characteristic ranges of variability for floating DO operation are synoptic variability (time scale variation is from day up to several day) and small-scale fluctuations (cyclicity from several seconds up to one hour). Complexity, non-uniform scale, polycyclicity, great variability of hydrometeorological processes result in necessity to consider them as stochastic functions of time and spatial coordinates and to describe their properties in terms of probabilistic characteristics. Further we shall understand the probabilistic model as the concrete kind of stochastic process record. It allows to obtain data on any probabilistic characteristics and, at the same time, to take into account dependence of the process of the factors included in conditions complex. These models are necessary for generalization of results of the analysis, compression of the information, an establishment of relation between various probabilistic characteristics and reproduction of realizations in non-observable situations [5].

Presence of multiscale variability causes a non-stationarity of hydrometeorological processes, and a variety of influencing factors (stratification of surface atmosphere layer and sea water, movement of baric formations and synoptic whirlwinds at oceans and seas) results in spatial heterogeneity. It results in necessity of using complex of several parametrically related probabilistic models in two time ranges for tool system.

For reproduction of spatial-time wave surface field $\zeta(x, y, t)$ relative to average level it is allowable to use a hypothesis about a stationarity and uniformity of the initial field first formulated by M.S. Longuet-Higgins [6]. However, the spectral model offered by him has not found wide application in real time systems due to low speed of convergence and essential computing expenses. As alternative we used model of a field of autoregress in the form [7]

$$
\zeta(x, y, t)=\sum_{i} \sum_{i} \sum_{k} \Phi_{i j k} \zeta(x-i, y-j, t-k)+\varepsilon(x, y, t)
$$

Here $\Phi_{\mathrm{ijk}}$ is the autoregress coefficients describing spatial-time connectivity of a field $\zeta(x, y, t), \varepsilon(x, y, t)$ is normal white noise with a dispersion $\sigma^{2}$, not dependent on $(x, y, t)$.

Parameters $\Phi_{\mathrm{ijk}}$ are determined by means of system of Yule-Walker equations through values of spatial-time correlation function $K_{\zeta}(x, y, t)$. However wave character of modeled surface motion (3) provides functional relation between spatial and time field components realizable as characteristics of the wave equation [8]. It allows to reduce regularity of initial problem setting model (3) by means of only spatial $S_{\zeta}(u, v)$ spectral density connected to correlation function by the ratio

$$
K_{\zeta}(x, y, t)=\iint S_{\zeta}(u, v) \cos (u x+v y+\omega(u, v) t) d u d v
$$

Here $u, v$ are the wave numbers inversely proportional to wavelength in two orthogonal directions, $\omega=\omega(\mathrm{u}, \mathrm{v})$ is the frequency determined by a dispersion relation kind of which depends on waveformation conditions. Alternative to (4) may be use of frequency - directed spectrum $S_{\zeta}(\omega, \Theta)$, connected with $S_{\zeta}(u, v)$ by means of nonlinear coordinates transformation [6].

Synoptic variability of waves is caused by formation, movement and disintegration of baric formations - cyclones and anticyclones accompanied with strong gradients of atmospheric pressure, and inducing alternation of storms and "weather windows". In 
the work [9] for the description of wave fields variability in storm the concept of climatic spectra, as probabilistic characteristics of time rows of functions $S_{\zeta}(u, v, \vec{r}, t)$ or $S_{\zeta}(\omega, \Theta, \vec{r}, t)$, dependent on spatial coordinates $\vec{r}$ and time $t$ is used. With a view of reduction of regularity let us consider a wave spectrum as the determined function of casual arguments $\Xi$ describing waveformation conditions :

$$
S(\omega, \Theta, \vec{r}, t)=S(\omega, \Theta, \Xi) . \quad \Xi=\Xi(\vec{r}, t)
$$

For example, in an affine vector $\Xi$ the following parameters may be included: average wind waves $h_{w}$ and swell $h_{s}$ height, average wind waves $\tau_{w}$ and swell $\tau_{s}$ period, average directions of wind waves $\Theta_{w}$ and swell $\Theta_{s}$ propagation, and also parameters of form, for example, peakness parameter $\gamma$ in JONSWAP approximation [10], also depending from wind speed $\vec{\nabla}(\vec{r}, t)$.

Thus, modeling of synoptic variability of spatial-time complex sea fields is reduced to reproduction of a casual affine vector field $\Xi(\vec{r}, t)=\left\{h_{\mathrm{w}}, \mathrm{h}_{\mathrm{s}}, \tau_{\mathrm{w}}, \tau_{\mathrm{s}}, \Theta_{\mathrm{w}}, \Theta_{\mathrm{s}}\right\}$. For this purpose in tool system the model of decomposition on orthogonal basis is used:

$$
\Xi(x, y, t)=\sum_{k} \alpha_{k}(t) \Psi_{k}(x, y)
$$

Here $\alpha_{k}(t)$ is the time-dependent scalar coefficients determining variability of field of parameters in time, $\Psi_{k}(x, y)$ are the affine basic functions (pair orthogonal). As optimum basis we use the natural orthogonal functions of an affine vector field determined as eigenfunctions of correlation nucleus through integral equation

$$
\int K_{\Xi}\left(\vec{r}_{1}, \vec{r}_{2}\right) \Psi_{k}\left(\vec{r}_{2}\right) d \vec{r}_{2}=\lambda_{k} \Psi_{k}\left(\vec{r}_{1}\right)
$$

Here $\lambda_{k}$ are eigennumbers of the equation; they characterize a variance of coefficients $\alpha_{k}(t)$.

Application of (6) for synoptic variability modeling of near-water wind speed is complicated by the fact that its volume $\vec{V}=(U, V)^{T}$ is a geometrical vector in Euclidian space: population mean $E[\bar{V}]$ is also a geometrical vector, and correlation function $K_{\nabla}\left(t, \tau, \vec{r}_{1}, \vec{r}_{2}\right)=E\left[\vec{V}\left(t, \vec{r}_{1}\right) \otimes \vec{V}\left(\tau, \vec{r}_{2}\right)\right]$ is a second rank tensor characterized by a set of invariants. In view of it, decomposition (6) becomes

$$
\vec{V}(x, y, t)=\sum_{k} \beta_{k}(t) \vec{\Psi}_{k}(x, y)
$$

where $\beta_{\mathrm{k}}(\mathrm{t})$ are scalar probabilistic processes, and $\Psi=\left(\Psi_{U}, \Psi_{V}\right)^{T}$ are vector natural orthogonal functions defined as the solution of homogeneous integral equations system in dyadic form

$$
\begin{aligned}
& \int K_{U U}\left(\vec{r}_{1}, \vec{r}_{2}\right) \Psi_{U}\left(\vec{r}_{2}\right) d \vec{r}_{2}+\int K_{U V}\left(\vec{r}_{1}, \vec{r}_{2}\right) \Psi_{V}\left(\vec{r}_{2}\right) d \vec{r}_{2}=\lambda \Psi_{U}\left(\vec{r}_{1}\right) \\
& \int K_{V U}\left(\vec{r}_{1}, \vec{r}_{2}\right) \Psi_{U}\left(\vec{r}_{2}\right) d \vec{r}_{2}+\int K_{V V}\left(\vec{r}_{1}, \vec{r}_{2}\right) \Psi_{V}\left(\vec{r}_{2}\right) d \vec{r}_{2}=\lambda \Psi_{V}\left(\vec{r}_{1}\right)
\end{aligned}
$$


Here $\mathrm{K}_{\mathrm{UU}}, \mathrm{K}_{\mathrm{UV}}, \mathrm{K}_{\mathrm{vU}}, \mathrm{K}_{\mathrm{vV}}$ are the appropriate components of correlation tensor. The eigenfunctions determined by this equation, as well as in (7), are orthogonal. They also provide the fastest convergence of decomposition (8) among all orthogonal bases with the square-law metrics.

Application of natural orthogonal basis $(7,9)$ in models $(6,8)$ allows to take into account the heterogeneity of initial field caused, for example, by special features of waveformation conditions (differences of depths, irregularity of coastal line, etc.), complex relief of land, and also by presence of ice cover on a part of water area. Such approach provides transition from model of casual fields $\Xi(\vec{r}, t), \vec{V}(\vec{r}, t)$ to model of time series $\left\{\alpha_{k}(t), \beta_{k}(t)\right\}$ of decomposition factors. In spite of the fact that for coefficients of decomposition on proper basis $\operatorname{cov}\left(\alpha_{k}\left(\mathrm{t}_{1}\right), \alpha_{j}\left(\mathrm{t}_{2}\right)\right)=0$ and $\operatorname{cov}\left(\beta_{k}\left(t_{1}\right), \beta_{i}\left(t_{2}\right)\right)=0$ at $t_{1}=t_{2}$ and $k \neq j$ are true, coefficients $\alpha_{k}(t)$ and $\beta_{k}(t)$ are correlated among themselves by virtue of functional dependence of wind and wave fields. For reproduction of system of related time series in tool system we use model of multivariate autoregression

$$
A_{t}=\sum_{k} \Phi_{k} A_{t-k}+E_{t}
$$

As against model of a scalar field (3), here $A_{t}=\left\{\alpha_{1}(t), \ldots, \alpha_{m}(t), \beta_{1}(t), \ldots, \beta_{n}(t)\right\}^{T}$, $E_{t}=\left\{\varepsilon_{1}, \ldots, \varepsilon_{m+n}\right\}^{\top}$ is the vector of correlated white noise, $\Phi_{k}=\left\{\phi_{i j k}\right\}$ are matrix coefficients of autoregression.

Taking into account, that it is possible to compare for each realization of variables $\{\Xi(\vec{r}, t), \vec{V}(\vec{r}, t)\}$ frequency- directed spectrum $(5)$, the problem of wind wave fields modeling in view of its synoptic and small-scale variability is reduced to reproduction of spectrum parameters (6), wind speed (8) with use of (10), and the subsequent generation of sea surface elevation field relative to average level in model ( 3 ) identified from relation (4).

In the fig. 3 by the Barents Sea example some probabilistic characteristics of wave and wind used for identification and verification of probabilistic models are shown: average wind speed hodograph and the main axes variance tensor per months (a), frequency (b) and frequency- directed (c) climatic spectra of the complex sea with the tolerant intervals determining their synoptic variability. Also in fig. 3 reproduction results of wind and waves modeling fields in synoptic range of variability (d), and three-dimensional wave fields in vicinities of point $(70 \mathrm{~N}, 40 \mathrm{E})$ are shown.
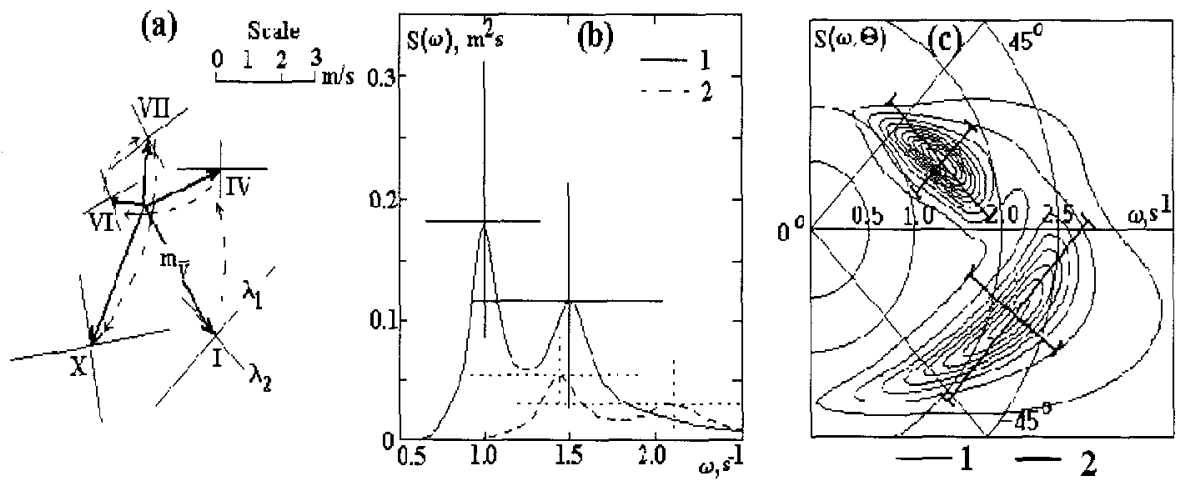

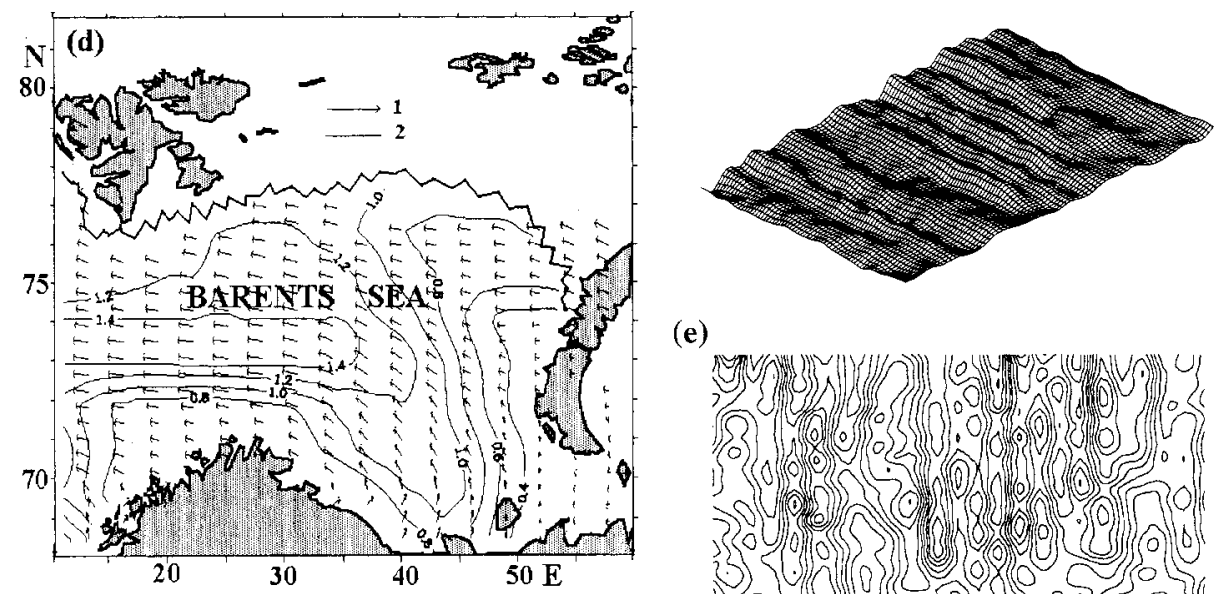

(e)

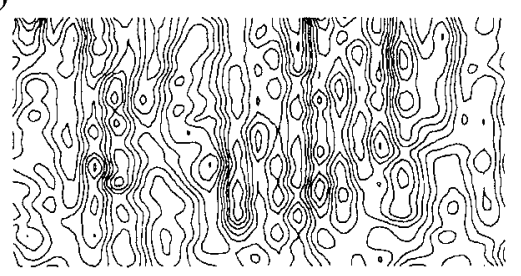

Fig. 3. Probabilistic characteristics of wind speed fields (a), wind waves (b,c), results of model calculations of wind and waves fields in synoptic range of variability (d) and in quasistationary range (e)

\section{Special features of "DO-environment" dynamics interaction}

Consideration of floating DO behavior under the wind and wave action is come to second Newton law:

$$
\sum_{i} \vec{F}_{i}=m \vec{a} ; \quad \sum_{i} \vec{M}_{i}=J \vec{\omega}
$$

where $m \vec{a}, J \vec{\omega}$ are the principal vector and moment of considered dynamic system. Components in right part could be divided, in general, into disturbing, restoring, inertial and damping components.

Many authors represent (11) as model systems of differential equations like

$$
F_{i}\left(x_{i}, \dot{x}_{i}, x_{i}, t, X_{i 1}, \ldots, X_{i m}, Y_{i 1}, \ldots Y_{i n}\right)=0,
$$

where $\mathrm{F}_{\mathrm{i}}(\bullet)$ are nonlinear functions; $\mathrm{x}_{\mathrm{i}}$ are linear and angular variables; $\mathrm{X}_{\mathrm{i} 1}, \ldots, \mathrm{X}_{\mathrm{im}}$ are the parameters describing DO as dynamic system (inertial, damping and restoring components); $\mathrm{Y}_{\mathrm{i} 1}, \ldots, \mathrm{Y}_{\mathrm{in}}$ are exciting forces and the moments; $\mathrm{t}$ is time; $\mathrm{i}=1,2, \ldots, 6$.

The experience shows that complication of model equations (12), terms improvement for disturbing actions and other components become ineffective at some moment. Terms' deriving is especially difficult in case of random actions, which arise from wind and waves.

Alternative to model equations is direct simulation of DO motion. Possibility of description of normal and transverse stresses distribution on DO wetted surface reduces simulation to clear procedure based on fundamental laws. It is necessary to 
solve Navier-Stokes problem alongside the DO hull and only potential problem can be solved at some from the hull (beyond the boundary layer). One of the main obstacles in the problem solution is unknown boundary (wave surface) where boundary conditions are satisfied. But the methods described in the previous chapter permit simulate 3D random sea waves in any time range. The character of proposed methods is physically adequate. So generated wave fields are in accord with hydrodynamics.

In this case we can divide complex problem into two simpler problems:

1. generation of time-spatial wave fields near floating DO

2. simulation of velocity and pressure fields in non-viscous fluid.

This solution is initial approximation to wave diffraction problem and NavierStokes problem in boundary layer.

Solution of potential problem is reduced to the following equation in the bottom hemisphere

$$
\begin{gathered}
\Delta \varphi=0 \\
\frac{\partial \varphi}{\partial t}+\frac{1}{2}\left(\left(\frac{\partial \varphi}{\partial x}\right)^{2}+\left(\frac{\partial \varphi}{\partial y}\right)^{2}+\left(\frac{\partial \varphi}{\partial z}\right)^{2}\right)+g \zeta=p \frac{\partial \zeta}{\partial t}+\frac{\partial \zeta}{\partial x} \frac{\partial \varphi}{\partial x}+\frac{\partial \zeta}{\partial y} \frac{\partial \varphi}{\partial y}=\frac{\partial \varphi}{\partial z}
\end{gathered}
$$

This is the linear problem with nonlinear boundary conditions and unknown boundary. But as we mentioned above methods of external actions modeling permit to reduce this problem. So the problem with unknown boundary becomes to problem with known boundary at each time step when two above mentioned boundary conditions are fulfilled. In this case we know process $\zeta$ in any space point and at the any time, and we can calculate any derivations in space and time domain using additional assumptions about wave nature (local wave numbers) [12]. Such way permits to obtain potential derivations on the wave surface and we can calculate them in any point in bottom hemisphere with the help of Newton potential theory.

Calculation of hydrodynamic pressure by means of Bernoulli's integral enables to calculate hydrodynamic forces and moments acting at the considered time moment.

$$
\vec{F}=-\int_{S_{0}} p \cdot \vec{n} d S \quad \vec{M}=-\int_{S_{0}} p \cdot(\vec{r} \times \vec{n}) d S
$$

where $S_{0}$ is the wetted ship surface, $n$ is a radius - vector of points of the wetted DO surface.

So the algorithm of DO motion simulation at sea could be proposed in the following form [11]:

1. Consequence of wave fields in considered region are generated $\zeta\left(x_{i}, y_{j}, t_{k}\right)$, where $\mathrm{i}=1 \ldots \ldots . . \mathrm{N} ; \mathrm{j}=1 . . \mathrm{M}\left(\mathrm{N} \times \mathrm{M}\right.$ - considered region), $\mathrm{t}_{\mathrm{k}}=\mathrm{k} \cdot \Delta \mathrm{t}, \mathrm{k}=0 . . \mathrm{L}$ (considered time moments)

2. All necessary wave characteristics are calculated.

3. Let us take any initial conditions for integration beginning. (At first moment we can assume that system pole coincides with centre of gravity)

The following items are fulfilled for each time step

4. The cross-points of wave and ship hull are found.

5. Pressure is calculated at wetted hull points.

6. Exiting force and moment components are calculated. 
7. Restoring forces and moments are calculated Submerged volume is calculated by integration.

Central of buoyancy is calculated too.

8. The system of all forces and moment is reduced to one main force and main moment (at first moment exciting force acts in the centre of gravity)

9. Let us integrate the system of differential equations at one time step. In this case such system has the following form that obtained from second Newton law:

$$
\begin{aligned}
& D \cdot X+F_{d}(X)=F \\
& J \cdot \ddot{\Phi}+M_{d}(\Phi)=M
\end{aligned}
$$

where $\mathrm{D}$ is DO mass, $\mathrm{X}$ is a linear displacement (surging, swaying or heaving), $\mathrm{J}$ is a correspondent DO inertia moment, $\Phi$ is an angle displacement (rolling, pitching, yawing), $F_{d}$ and $M_{d}$ are the correspondent damping forces and moments, $F$ and $M$ are the components of main force and main moment.

10.Momentary centre of motion is obtained. This is the point of excitation forces action at the next time step.

\section{Results of experiments}

The IS full-scale tests have been carried out aboard a tanker in the Baltic Sea, on a container ship in a voyage in the Mediterranean and in the Atlantic, on a small ship in the Black Sea.

The test proved the possibility of practical evaluation and prediction of the dynamic characteristics, wind waves, the reliability of $\mathrm{KB}$ operation under various conditions of service. KB was design in accordance with preliminary situation simulation. For these purposes hydrodynamic and probabilistic modeling of wind waves and ship motion were fulfilled.

The table shows the comparative data about the different ways of measuring seas parameters obtained at the containership. In the table you can sce that the IS method applied to the seas estimation on the basis of identification method gives good results which correspond to the actual measurement data received by means of standard wave-meters and systems (string wave recorder, laser sensor, wave recorder GM-32).

Table 1. Wave height measurement (3\% quantile, $\mathrm{m}$ )

\begin{tabular}{|l|l|l|l|l|l|}
\hline $\begin{array}{c}\text { Wave force } \\
\text { (balls) }\end{array}$ & $\begin{array}{c}\text { Laser } \\
\text { sensor }\end{array}$ & $\begin{array}{c}\text { Wave } \\
\text { recorder }\end{array}$ & $\begin{array}{c}\text { String wave } \\
\text { recorder GM-32 }\end{array}$ & $\begin{array}{c}\text { Designed } \\
\text { algorithm }\end{array}$ & $\begin{array}{c}\text { Max } \\
\text { Div. \%, }\end{array}$ \\
\hline 3 & - & - & 1,17 & 1,23 & 5,1 \\
\hline 4 & 1,80 & 1,74 & 1,76 & 1,85 & 6,3 \\
\hline 5 & 3,28 & 3,17 & - & 3,40 & 7,2 \\
\hline 6 & 5,72 & 5,61 & - & 5,83 & 3,9 \\
\hline
\end{tabular}




\section{Acknowledgement}

The work is supported by grants INTAS Open 1999 - N666 and RFBR 00-07-90227

\section{References}

1. Nechaev Yu.I., Degtyarev A.B. Account of peculiarities of ship's non-linear dynamics in seaworthiness estimation in real-time intelligence systems. Proc. of the $7^{\text {th }}$ International Conference on Stability of Ships and Ocean Vehicles. Launceston, Tasmania, Australia, February 2000, vol.B, pp.688-701.

2. Nechaev Yu.I., Degtyarev A.B., Boukhanovsky A.V. Analysis of extremal situations and ship dynamics in seaway in intelligent system of ship safety monitoring. Proc. of the $6^{\text {th }}$ International Conference on Stability of Ships and Ocean Vehicles. Varna, Bulgaria, September 1997, vol.I, pp.351-359.

3. Boukhanovsky A.V., Degtyarev A.B. The instrumental tool of wave generation modelling in ship-borne intelligence systems. Proc. of $3^{\text {td }}$ International Conference CRF-96, St.Petersburg, Russia, June 1996, vol.I, pp.464-469

4. Nechaev Yu.I., Degtyarev A.B., Boukhanovsky A.V. Adaptive forecast in real-time intelligence systems. Proc. of $13^{\text {th }}$ International conference on hydrodynamics in ship design HYDRONAV'99, Gdansk-Osroda, Poland, September, 1999, pp.229-235.

5. Lopatoukhin L.J., Rozhkov V.A., Bukhanovsky A.V. The main problems of wind and wave statistics, based on spectral modelling data. Proc. of Coastal Wave Meeting, September, 25-28, Barselona, Spain, 2000, paper 7.5.

6. Longuet-Higgins M.S. The statistical analysis of a random moving surface. Phil. Trans. Roy. Soc., London, 1957, 249, N966, pp.321-387

7. Rozhkov V.A., Trapeznikov Yu.A. Probability models of oceanological processes. Leningrad, Gidrometeoizdat P.H., 1990 (in Russian)

8. Komen G.L., Cavaleri L., Donelan M., Hasselmann K., Hasselmann S., Janssen P. Dynamics and modelling of ocean waves. Cambridge University Press., 1994.

9. Boukhanovsky A.V., Lopatoukhin L.J., Rozhkov V.A. Wave climate spectra and wave energy resources in some Russian seas. WMO/TD - No 938 "Provision and engineering/operational application of ocean wave data", 1998, pp.324-333.

10. Hasselmann K. et al. Measurements of wind-waves growth and swell decay during the Joint North Sea Wave Project (JONSWAP). - Hamburg: Deutsch. Hydrogr. Inst., 1973.

11. Degtyarev A.B., Podolyakin A.B., Imitative modelling of ship behaviour in random sea. Proc. of International shipbuilding conference'98, St.Petersburg, Russia, November 1998, vol.B, pp.418-426. (in Russian)

12. Whitham G.B. Linear and nonlinear waves. - New York: John Wiley \& Sons, 1974 